\title{
La reseña de formación: propuesta de modelo analítico
}

The training book review: analytical model proposal

\author{
Alba Naroa Romero González \\ Instituto IES Ibarrekolanda \\ España
}

\section{Manuela de las Nieves Álvarez Álvarez}

Universidad de Deusto

España

\section{(c) $(\mathrm{i}) \bigodot_{\mathrm{BY}}$}

Alba Naroa Romero González: Departamento de Lengua Castellana y Literatura, Instituto IES Ibarrekolanda, España. | E-mail: alromer@opendeusto.es

Manuela de las Nieves Álvarez Álvarez: Departamento Lenguas Modernas y Estudios Vascos, Facultad de Ciencias Sociales y Humanas, Universidad de Deusto, España. | E-mail: manuela.alvarez@deusto.es 


\section{Resumen}

Este artículo se centra en un género poco atendido, como es la reseña académica de formación, con el objetivo de analizar su configuración retórico-lingüística siguiendo el planteamiento de tradición swalesiana de análisis de género. En este marco, se realiza un estudio empírico de corte mixto que analiza un corpus de 96 reseñas producidas en español por la población de estudiantes universitarios de un programa de formación completo como es el grado en Lenguas Modernas (Universidad de Deusto). En este corpus producido a efectos de la investigación, se identifican y analizan los propósitos comunicativos de las reseñas (movimientos retóricos) y los objetivos comunicativos de menor rango que completan esos propósitos (pasos y subpasos). A partir de este análisis, se crea y se valida un modelo analítico que se articula en dos movimientos retóricos (describir y valorar) para las reseñas académicas de formación. Este modelo se presenta como una herramienta útil para valorar la progresión de los estudiantes en la selección y organización de la información y en el desarrollo de la descripción y la argumentación.

Palabras clave: estructura retórico-discursiva; reseña académica de formación; análisis de género; escritura académica; estudiantes universitarios.

\section{Abstract}

This research article is about a genre almost ignored by the researchers as the training book review. The purpose of the study is to analyse its rhetorical-linguistic structure using genre analysis in the spirit of Swalesian tradition. In this frame, this paper presents an empirical qualitative research, in which 96 Spanish training book reviews, produced by the population of students from the complete degree of Modern Languages, are analysed. In this corpus produced for the study, higher level communicative purposes (rhetorical moves) and lower level communicative purposes (steps and substeps) from these training book reviews are identified and analysed. This model is a useful tool to assess the progression of students in selecting and organising information and in the development of the description and the argumentation.

Keywords: rhetorical-linguistic structure; training book review; genre analysis; academic writing; university students. 


\section{Introducción}

En las últimas décadas, el interés por identificar y caracterizar los géneros académicos según las disciplinas o la lengua de uso ha aumentado exponencialmente (Babaii y Ansary, 2007). Este interés se ha intensificado, además, en relación con los géneros que producen los estudiantes en el proceso de aprendizaje para adquirir los conocimientos disciplinares y como medio para ingresar en una cultura disciplinar (Hyland, 2007; Camps y Castelló, 2013).

Entre esta variedad de géneros para aprender, la reseña, reconocida como tal desde la década de los 90 (North, 1992; Motta-Roth, 1998; Gea-Valor, 2000; De Carvalho, 2002; Díaz-Blanca, 2015), es uno de los más frecuentes (Alzari, D’Alessandro y Radiminski, 2014). Es considerado un género facilitador del aprendizaje de los conocimientos disciplinares (Alzari, 2012), ya que, tal y como señala Hammond (2011), un género de aprendizaje permite al estudiante conocer los modos de interpretar y construir el conocimiento disciplinar en un contexto social concreto. Este tipo de reseña, producida por los estudiantes para aprender a leer y escribir en la universidad, se denomina reseña académica de formación (Alzari, D’Alessandro y Radiminski, 2014).

No obstante, pese a su recurrencia en las aulas universitarias y su funcionalidad como género para aprender, la investigación sobre este género no es muy abundante y suele centrarse en la estructura retórica de reseñas producidas por expertos (Motta-Roth, 2001; De Carvalho, 2002; Navarro, 2011), y no tanto en la de reseñas producidas por estudiantes (Alzari, 2012). Tampoco se ha indagado acerca de cómo la reseña de formación puede contribuir al desarrollo del perfil investigador del estudiante mediante los procesos de construcción del conocimiento que implica (análisis crítico e interpretación de textos, selección y organización conceptual de los contenidos, diálogo entre miembros de una cultura disciplinar, posicionamiento del autor, etc.). Este vacío en cuanto a la investigación acerca de las reseñas académicas de formación conduce a una teorización poco definida sobre estas que se fundamenta en la observación (Alzari, D’Alessandro y Radiminski, 2014), no en estudios empíricos que aporten instrumentos para valorar las transformaciones de las producciones de los estudiantes y evalúen su progreso.

El estudio que aquí se presenta se propone caracterizar el género reseña académica de formación mediante la metodología de análisis de género (Swales, 1990, 2004) para, así, crear y validar un modelo analítico de la estructura retórica de este género. Desde este modelo validado y creado según el nivel de experticia del estudiante, el docente podrá evaluar la adquisición por parte del estudiante de habilidades retórico-discursivas que le permitan participar en la cultura disciplinar.

En los siguientes apartados, se presentan los conceptos que sirven de andamiaje teórico para este estudio (la reseña académica de formación y el análisis de género), los aspectos relativos a la metodología de esta investigación (informantes y corpus, instrumento y procedimiento de análisis), los resultados y su discusión y, por último, las conclusiones y las implicaciones que estas pudieran tener en la enseñanza de este género discursivo. 


\section{Marco teórico}

\subsection{La reseña académica de formación}

La reseña académica de formación es un género producido por los estudiantes para favorecer su aprendizaje mediante la interpretación y el análisis crítico de una obra (Giammatteo y Ferrari, 2000), mientras que la reseña académica experta es producida por académicos con el objetivo de dar cuenta crítica de las novedades bibliográficas para facilitar que los investigadores encuentren novedades, validar el conocimiento científico e intercambiar opiniones (Gea-Valor, 2000; Groom, 2009; Giannoni, 2009). Ambas se definen según su contexto de producción (tarea de aula o publicación científica), el autor (estudiante o académico) y el objetivo didáctico de la tarea (Navarro y Abramovich, 2012).

Este es un género de gran importancia para que los estudiantes reflexionen sobre las estrategias retórico-discursivas del texto, las convenciones de cada cultura disciplinar y, por supuesto, otro tipo de cuestiones de tipo idiomático; esto es, contribuye a que se familiaricen, precisamente, con la estructura, el registro, el uso de la voz, la argumentación y el estilo de los géneros académicos (Ferrari, 2005), los cuales se entienden siempre como prácticas sociales delimitadas por la cultura disciplinar en la que se producen (Hyland, 2014). Así, al existir diferencias entre las reseñas que se producen en diferentes disciplinas, este género sirve también para que los estudiantes aprecien las diferencias en la construcción del conocimiento en un mismo género entre culturas disciplinares (Motta-Roth, 1996; Díaz-Blanca, 2015).

Por consiguiente, la reseña académica de formación es una herramienta útil en los inicios de la alfabetización académica para que los estudiantes universitarios:

- se inicien en el desarrollo de la escritura académica mediante la interpretación de otros géneros académicos que van a ser reseñados y la interpretación de sus contenidos para adoptar una postura crítica en la producción de la reseña;

- conozcan otros géneros académicos de mayor complejidad que les puedan servir como fuentes bibliográficas (manuales, artículos de investigación) o como modelos para llevar a cabo actividades de escritura más largas y complejas de ámbitos disciplinares diversos que tendrán que producir a medida que avancen los cursos (ensayos, monografías);

- ingresen y participen de manera activa y progresiva en la comunidad discursiva en la que se estén formando al conocer estrategias retóricas, discursivas y lingüísticas propias de esa cultura disciplinar.

No obstante, a pesar de las potencialidades de este género como un género de aprendizaje, este ha recibido escasa atención por parte de los investigadores debido al bajo índice de citación y, por ende, a la poca significatividad para el currículum del autor que la realiza (Motta- 
Roth, 1996; Alcaraz y Salager-Meyer, 2005), y esa atención se ha focalizado, además, en textos producidos por expertos. Es la definición de Motta-Roth (1995) de la reseña en la década de los 90 desde los fundamente teóricos del análisis de género de tradición swalesiana la que arroja luz sobre la necesidad de su estudio al definirla a través de los tres elementos clave de la definición del género de Swales (1990): (1) es un evento comunicativo porque se produce en un contexto social concreto con el propósito de evaluar un libro; (2) es un evento comunicativo reconocido por la comunidad académica; y (3) responde a las expectativas del lector del texto.

\subsection{El análisis de género}

El análisis de género, también conocido como análisis de la estructura retórica del texto, parte originalmente de visiones del género arraigadas en la teoría de los enunciados de Bajtín (1982). De ahí surge el enfoque funcional de Swales (1990), que es aquel que se preocupa por la estructura retórica del texto, por los propósitos comunicativos que dan estructura al género.

El análisis de género es una teoría útil para identificar y caracterizar los géneros en su contexto. Desde que Swales (1990) caracteriza la introducción del artículo de investigación en tres propósitos comunicativos (establecer el territorio, establecer el nicho y ocupar el nicho), el análisis de género se convierte rápidamente en uno de los tipos de análisis de la estructura retórica del texto más importantes y acusados. Además, con la ampliación de Bathia (1993), quien propone aplicarlo para el análisis de todo tipo de géneros académicos y profesionales (Gea-Valor, 2000: 13), este empieza a cobrar cada vez más fuerza, de manera que han proliferado gran abundancia de publicaciones que analizan la estructura retórica de los géneros académicos institucionalizados que producen los autores expertos (Maswana, Kanamarub y Tajinob, 2015; Salmani y Montazeran, 2012; Al-Khasawneh, 2017), pero también aquellos que producen los estudiantes (Ding, 2007; Pramoolsook y Qian, 2013), con especial atención en la última década a los trabajos fin de grado (Zamora y Venegas, 2012; Velasco, 2017) y los trabajos fin de máster (Samraj, 2008).

Esta teorización, como se ha demostrado en la variedad de estudios Ilevados a cabo bajo su ala, da cuenta de las diferencias entre distintos géneros, entre los mismos géneros producidos en disciplinas diferentes y en la misma disciplina según el contexto de producción del texto y el nivel de experticia del autor. Asimismo, ha dado cuenta de que los géneros más institucionalizados presenten menor libertad creativa para el autor, exigiéndole que respete unas normas y nomenclaturas (Kanoksilpatham, 2007).

Es, precisamente, esta dicotomía de género y contexto lo que convierte el método de análisis de género en una herramienta útil para valorar las transformaciones de la escritura académica de los estudiantes a lo largo de la carrera, mientras que evaluar los géneros que producen estos estudiantes que recién ingresan en la cultura disciplinar tomando como referente aquellos que producen los expertos que están inmersos en ella es un error. Por consiguiente, 
conocer verdaderamente las estrategias retórico-discursivas y lingüísticas que emplean los estudiantes implica analizar los géneros que ellos producen en su contexto para, desde su praxis, abordar la alfabetización académica.

Desde esta teorización, se manejan los movimientos retóricos (move) o MR, que se refieren a segmentos de texto que cumplen una función u objetivo comunicativo específico que contribuye a la estructura retórica global del texto, para lo cual reúne unas características estructurales y sintácticas específicas (Swales, 2004; Sabaj, Toro y Fuentes, 2011; Piqué-Noguera y Camaño-Puig, 2015). Su estudio lleva necesariamente al análisis de los pasos (steps) que son subfunciones u objetivos comunicativos específicos que contribuyen al desarrollo de cada movimiento retórico (Swales, 2004; Diafabio de Anglat y Álvarez, 2019) y estas subfunciones pueden estar a su vez conformadas por subpasos que las dotan de significado (Venegas, Zamora y Galdames, 2016). Cabe destacar que no es fácil delimitar un paso respecto a otro, ni un subpaso respecto a otro, ya que pueden ocupar parte de una oración, una oración entera, varias o incluso párrafos (Sabaj, 2012; Piqué-Noguera y Camaño-Puig, 2015).

\section{Marco metodológico}

Este es un estudio empírico de carácter descriptivo y exploratorio basado en un corpus cuyo objetivo, como ya se especifica en la introducción, es caracterizar el género reseña académica de formación.

\subsection{Participantes y corpus}

El corpus de este estudio está compuesto por 96 reseñas académicas de formación en español producidas ad hoc por toda la población de estudiantes universitarios de nueva matrícula de los cuatro cursos del grado en Lenguas Modernas (LLMM) de la Universidad de Deusto (Bilbao, País Vasco) durante el curso 2015/2016. En consecuencia, la población que participa en este estudio resulta ser el conjunto de estudiantes de todo un programa formativo.

\subsection{Instrumento}

El primer paso para diseñar el instrumento ha sido seleccionar el objeto de la reseña de formación. En este respecto, se toman tres decisiones importantes para la búsqueda: el libro debe estar en castellano; la obra debe pertenecer a la disciplina de estudio, de manera que contribuya a la creación de un espacio epistémico de la lingüística; debe tener una portada, contraportada, índice y presentación que les aporte a los autores de las reseñas toda la información que necesitan para construir el texto de manera espontánea. Con esos criterios, se selecciona, finalmente, la Gramática didáctica del español (Gómez, 1998).

Seleccionada esta obra, se pasa a diseñar la consigna, proponiendo una situación comunicativa a la que los estudiantes deben dar respuesta: la biblioteca de la Universidad de Deusto 
quiere incorporar una reseña de los libros de reciente incorporación en su catálogo, para lo que solicita a los estudiantes de la universidad un texto de, al menos, 200 palabras que ayude a los usuarios de la biblioteca en su elección.

Elaborado el instrumento, se pone a prueba en un pretest con los estudiantes de segundo del grado en Lenguas Modernas y Gestión por su formación en común con los participantes del estudio. Este pretest proporciona información relevante respecto a los contenidos y la redacción para la mejora del instrumento que se aplica finalmente en las aulas del grado en LLMM (ver instrucción en el anexo). La aplicación se lleva a cabo en el marco de materias disciplinares que cursan los estudiantes. Estos, tras conocer toda la información relativa al estudio (el cual se enmarca en una tesis doctoral cuyo objetivo es caracterizar la escritura académica de los estudiantes de grado a través de su trayectoria), reciben la instrucción, las fotocopias de la portada, la contraportada, el índice y la presentación del texto fuente, y el papel para llevar a cabo la actividad en el aula.

\subsection{Procedimiento de análisis}

El análisis de la estructura retórica de las reseñas se lleva a cabo siguiendo las pautas del análisis de género. Este procedimiento se divide en dos fases: pre-análisis y análisis.

En la fase de pre-análisis, se sigue la metodología de análisis BCU (Biber, Connor y Upton, 2007) articulada en siete pasos: identificar los propósitos comunicativos globales; segmentar el texto en unidades temáticas; clasificar las unidades temáticas en categorías; definir los criterios de inclusión y exclusión de cada tipo de unidad temática según criterios léxico-gramaticales; definir cada categoría según las unidades temáticas que la componen; analizar los textos completos en busca de secuencias; e identificar modelos de organización del texto.

Este procedimiento de análisis conduce a un modelo analítico de una estructura global de dos movimientos retóricos desplegados en diferentes pasos y subpasos que es preciso validar. Con ese propósito, se lleva a cabo un juicio de expertos que valora la confiabilidad del modelo. Los jueces que participan en la validación son cinco expertos doctores en lingüística, especializados en áreas relacionadas con la escritura académica, el análisis de género y la creación de instrumentos de análisis.

Esta valoración se realiza de manera individual, mediante correo electrónico. Todos los expertos reciben un texto informativo sobre la investigación en la que se enmarca este estudio, los documentos con la instrucción y las fotocopias del libro que reseñaron los informantes, y un tercer documento con el modelo de análisis y las instrucciones para evaluarlo. Realizan dos tipos de evaluación: (1) acerca de la pertinencia de cada paso y subpaso a los movimientos retóricos que se han identificado, y (2) acerca de la claridad, la coherencia y la relevancia de cada paso y subpaso en una escala del 1 al 4 . Adicionalmente, también pueden dejar observaciones sobre cada paso y subpaso. 
Una vez recibidas las respuestas de los expertos, se calcula el porcentaje de acuerdo entre los expertos mediante el coeficiente de concordancia $W$ de Kendall en el programa de análisis cuantitativo SPSS. Los resultados del cálculo de este coeficiente descartan la hipótesis nula, y se logra un rango de acuerdo interjueces de 0.79 .

Por otro lado, los expertos realizan una serie de observaciones cualitativas que permiten reajustar la formulación de los pasos y los subpasos. Entre estos reajustes de mejora del modelo analítico destaca la definición de los MR, pasos y subpasos con verbos en 3. a persona del singular del presente de indicativo en lugar de usar nominalizaciones, como inicialmente se había hecho, para evitar su confusión con categorías de contenido. Otras modificaciones, hechas a partir de los comentarios de los expertos, se refieren a reformulaciones de algunas expresiones que no les resultaron a los expertos suficientemente comprensibles y en cuyas observaciones hubo un acuerdo entre ellos.

Finalizado este procedimiento de análisis, se obtiene un modelo analítico de los movimientos retóricos de las reseñas académicas de formación que comprende los MR, los pasos y los subpasos que se despliegan en la configuración del género estudiado.

\section{Resultados y discusión}

El modelo analítico de la reseña académica de formación resultante del análisis de este corpus confirma la existencia de dos únicos movimientos retóricos: describir y valorar. Cabe destacar, antes de descender al análisis pormenorizado de cada MR, que, pese a que se han identificado estos dos propósitos comunicativos en la totalidad del corpus, no todos los informantes se han valido para el desarrollo de la tarea de ambos simultáneamente, lo que ha resultado en textos en los que solo se describe o solo se valora.

Como cada uno de estos movimientos retóricos tiene su propia caracterización y un despliegue en pasos y subpasos diferente, se presenta a continuación los resultados obtenidos del análisis de cada MR por separado.

\subsection{M1: Describe el libro objeto de la reseña}

En el 97,9\% de las reseñas analizadas aparece, en al menos una ocasión, el movimiento retórico denominado Mz. En el Mz, describir el libro, el propósito comunicativo es doble: (1) establecer el marco general de la obra y (2) definir la estructura de los contenidos del libro. Hay que puntualizar que, aunque este se ha caracterizado como el primer propósito de la reseña, no siempre se sigue este orden. Es decir, se presentan reseñas en las que el Mı aparece después del M2 o el M1 se presenta de manera recursiva. Sin embargo, se ha caracterizado este movimiento retórico como el primero porque este es el que se presenta en la mayor parte de las reseñas en primer lugar (en el 91,6\% de los casos). 
Para el desarrollo de este propósito global, el Ml, se despliegan un total de nueve pasos con sus correspondientes subpasos en cada caso. La tabla 1 muestra los pasos y subpasos identificados en el MI. En esta, los segmentos textuales se han codificado utilizando "P" para los pasos y un número identificativo y "SP" para los subpasos añadiendo el número del paso en el que se encuadran y una letra sucesiva. Así, pasos como el P1, el P2, el P6 y el P8 se despliegan también en una variedad de subpasos (SP1A, SP1B, SP1C, SP2A...).

\section{TABLA 1}

Modelo de análisis de los movimientos retóricos en reseñas de formación

\section{M1: DESCRIBE EL LIBRO}

\section{P1: presenta al autor del libro}

SPIA: Indica el nombre y los apellidos del autor

SPıB: Identifica la titulación académica del autor

$\mathrm{SP}_{1} \mathrm{C}$ : Identifica el cargo profesional del autor

\section{P2: describe el contexto de producción del libro}

SP2A: Indica los datos editoriales del libro

SP2B: Identifica el número de edición del libro

SP2C: Indica el número de ISBN

SP2D: Indica el año de publicación del libro

SP2E: Identifica otras figuras además del autor principal al cargo

de la obra (coordinadores, supervisores, diseñadores...)

\section{P3: especifica el tipo de libro}

P4: identifica el o los objetivos del libro

\section{P5: identifica el o los temas del libro}

\section{P6: describe la organización del libro}

SP6A: Señala el número de capítulos que componen el libro

SP6B: Señala los títulos de todos los capítulos que componen el libro

SP6C: Selecciona algunos contenidos de manera arbitraria

SP6D: Identifica la estructura del libro a través de los contenidos de los capítulos

\section{P7: identifica el enfoque teórico}

P8: se refiere a otras características del libro

SP8A: Indica los recursos que facilitan la comprensión del contenido del libro al lector

SP8B: Especifica los recursos que sirven para realizar síntesis conceptuales y temáticas

SP8C: Alude a cuestiones de diseño y formato del libro

\section{P9: localiza físicamente el libro en uno o varios lugares}


Hay que puntualizar, además, que no en todos los pasos ha sido clara la diferenciación entre el Mı y el M2. Este sería, por ejemplo, el caso del P8, en el cual es muy difusa la línea que separa la descripción de la valoración. En este paso se aportan datos que pudieran conducir a pensar que se trata de una valoración de la calidad del libro. Por esa razón, se toma la decisión de que formará parte del P8 del Mı cuando los datos no estén acompañados de estructuras gramaticales propias de la argumentación que denotan valoración (verbos dicendi, verbos de causa-consecuencia, construcciones con "se", conectores lógicos de causa-consecuencia, operadores argumentativos, etc.) y que, por el contrario, formará parte del P1 del M2 (se verá en el próximo apartado) cuando estén explícitamente acompañados de estructuras gramaticales propias de la argumentación.

Después de estas aclaraciones iniciales, se procede a presentar cada uno de los pasos que constituyen el Mı en este modelo analítico de la reseña académica de formación. En el primer paso identificado, el $\mathrm{P} 1$, que es además el más recurrente de este corpus con un porcentaje del 92,7\%, se presenta al autor del libro con tres propósitos: identificarlo como individuo, identificar su titulación académica e identificar su cargo profesional. De estos objetivos, los dos últimos aportan información que sirve para que el lector, mediante la trayectoria del autor, defina su idoneidad sobre esa temática. Por ejemplo:

(1) La biblioteca de Deusto [sic] ha puesto a disposición el nuevo libro de Leonardo Gómez Torrego, Doctor en filología [sic] y [sic] investigador del CSIC. (Informante 1.8)

El segundo paso, el P2, lo emplea el 21,8\%. Aquí se contextualiza el libro en base a diferentes aspectos de su propia creación con información que va desde la fecha de publicación, la editorial que lo hace posible, la edición del libro o el año de publicación hasta referirse incluso a cualquier individuo que haya tomado parte en la creación del libro además del autor (coordinadores, supervisores...). Véase el siguiente ejemplo:

(2) La reseña que expongo a continuación es sobre “Gramática didáctica del español”, libro escrito por Leonardo Gómez y que pertenece a la editorial SM. A parte [sic] de Leonardo Torrego (quien ha sido el autor del libro), hay también más gente que ha colaborado en la edición y supervisión como es el ejemplo de Concepción Maldonado, la cual es Doctora en Filología Hispánica.

No es la primera edición que se hace de este libro, la versión actual está corregida y aumentada. (Informante 1.25)

Del tercer paso al séptimo, a diferencia del P1 y el P2 y de los posteriores P8 y P9, todos los pasos se refieren de forma explícita a los contenidos del libro. Para empezar, en el tercero, el P3, que es el menos frecuente con un porcentaje de recurrencia del 1\%, el objetivo es identificar a qué género o tipología textual pertenece el libro (manual, ensayo, guía, etc.). Como, por ejemplo:

(3) Gramática didáctica del español, de Leonardo Gómez Torrego, es un manual de gramática en el que la información pretende ser transmitida de manera sencilla al lector, sin renunciar a la eficacia. (Informante 4.75) 
La línea divisora entre el cuarto y el quinto paso resulta más difícil de trazar, ya que uno puede estar implícito en el otro. Por esa razón, se han diferenciado los pasos por lo que se dice explícitamente. Es decir, para que sea considerado objetivo del libro, el P4, se tiene que formular en infinitivo o hay que presentarlo especificando que se trata del "objetivo", "propósito", "finalidad" o cualquier posible sinónimo. En el caso del tema, el P5, se debe especificar que se está hablando del "tema" del libro o introducirlo con otras fórmulas que induzcan a interpretarlo como el tema ("es sobre”, "acerca de”; "trata de”; "basado en”...). Entre estos dos, los informantes suelen decantarse más por el P5 en el 54,1\% de los casos mientras que el P4 aparece escasamente en el 28,1\%. Ahora bien, los informantes, generalmente, no desarrollan ambos pasos simultáneamente en la misma reseña. Algunos ejemplos ilustrativos:

(4) Este libro, "Gramática didáctica del español”, ha sido escrito por el Doctor en filología [sic] Leonardo Gómez Torrego, con el objetivo de facilitar el entendimiento del español y su gramática. (Informante 2.53)

(5) (...) es un libro didáctico organizado en diferentes secciones que tratan sobre la gramática española. (Informante 2.47)

En el sexto paso, el P6, se describe la organización del libro, para lo que se puede recurrir desde citar el número de capítulos que componen el libro o copiar los títulos de esos capítulos hasta referirse a algunos contenidos de manera arbitraria o identificar cómo se estructura el libro mediante los contenidos de los capítulos. A este paso se recurre frecuentemente con un nivel de recurrencia del 66,6\%. Véase el siguiente ejemplo:

(6) La organización de los contenidos está dividida en capítulos que siguen un orden en función a su grado de dificultad. En primer lugar, encontramos una breve introducción que precede a los capítulos. Tras la introducción, se abordarán las clases de palabras en el primer apartado, donde podremos examinar el sustantivo, el adjetivo, los determinativos, los pronombres, los verbos, los adverbios, las preposiciones, las conjunciones y las interjecciones. El segundo capítulo, cuya extensión es más limitada, estará dedicado a las oraciones, los enunciados y los grupos sintácticos. Dicho apartado estará segmentado en tres subapartados: el primero estará dedicado a los enunciados y las oraciones, el segundo a los grupos sintácticos y el tercero a oraciones y conjuntos. Los últimos dos capítulos, fonética y fonología; y ortografía, están [sic] estrechamente relacionados y estarán [sic] orientados hacia las partes de la gramática que suelen entrañar mayor dificultad. (Informante 3.66)

En el último paso que se refiere a los contenidos del libro, el P7, se identifica el enfoque teórico. Esto es, se distingue la corriente, enfoque o perspectiva desde la que se ha escrito el libro y, por tanto, desde la cual debe ser interpretado. Solo es habitual en el 32,3\% del corpus. Por ejemplo:

(7) Este libro ofrece un enfoque de la gramática del español descriptivo y normativo, de un modo fácil. (Informante 4.81) 
El P8, aunque sí se fija en aspectos del libro que están en su interior, no se detiene en los propios contenidos del libro. Lo que le interesa es qué recursos facilitan la comprensión de esos contenidos (ejercicios resueltos, ejemplos, claridad y sencillez de la exposición de los contenidos, pertinencia de la selección de los contenidos...), qué recursos sirven para realizar síntesis conceptuales y temáticas (cuadros de norma, remisiones, correspondencias terminológicas, llamadas de atención, notas aclaratorias, el índice temático...) y cuál es el diseño y formato del libro (diseño de páginas, tamaño de los párrafos, número de páginas, atractivo visual del libro...). A este paso los informantes recurren con bastante frecuencia en el $72,9 \%$ de los casos.

(8) Este libro de gramatica [sic] expone los contenidos con sencillez y de [sic] una gran cantidad de ejemplos por [sic] así facilitar la lectura.

Además cuenta con llamadas de Atención [sic] con los [sic] que se recuerdan los errores comunes que se cuentan [sic] en la lengua española.

Cuenta con una estructura clara y sencilla, con párrafos [sic] cortos y explicaciones extra al final de cada unidad. También cuenta con ejercicios, con soluciones, por [sic] contrastar lo que se ha aprendido.

Para enriquecer el libro se han introducido aspectos normativos para reflexionar sobre varios aspectos como: laísmos [sic], dequeísmos [sic] etc. (Informante 4.76)

Por último, el noveno paso, el Pg, se refiere a la localización física del libro. Es decir, se identifica un lugar concreto en el que se puede encontrar ese libro. Su recurrencia es escasa, prácticamente residual, con el 2,1\%.

(9) Este manual se encuentra en la biblioteca de la universidad [sic] de Deusto. Este libro es uno de los nuevos incorporados en el catálogo de la biblioteca. En la secretaria de la biblioteca se ofrece ayuda para la búsqueda [sic] de cualquier libro, de lo contrario el manual de gramática española se encuentra en el tercer piso junto con otros libros en el ámbito lingüístico. (Informante 2.56)

Ahondando más en estos resultados, el cálculo de la recurrencia de cada uno de estos pasos permite identificar qué pasos utilizan los informantes de manera obligatoria (más del $66,6 \%$ de los informantes), de manera opcional (entre el 33,3\% y el 66,6\% de los informantes) o de manera poco frecuente (menos del 33,3\% de los informantes). De esta manera, se constituirían como pasos obligatorios el P1 (presenta al autor del libro), el P8 (se refiere a otras características del libro) y el P6 (describe la organización del libro) mientras que solamente se presentaría de manera opcional el P5 (identifica el o los temas del libro). Los cinco pasos restantes del Mı tienen una presencia muy inferior que, en algunos casos (el P9 y el P3), es prácticamente residual.

\subsection{M2: valora el libro objeto de la reseña}

En el 90,6\% de las reseñas analizadas aparece el movimiento retórico que se ha denominado M2. En el M2, valorar el libro, el propósito comunicativo es también doble: (1) valorar de mane- 
ra positiva o negativa diferentes aspectos del libro y (2) recomendar o no la obra al lector. Pese a que ocupa en la mayor parte de los casos el segundo lugar en cuanto al orden de aparición en la reseña, hay que señalar que se presentan algunos casos de reseñas que primero valoran y, luego, describen el libro (el 8,4\%).

Para el desarrollo de este propósito global se despliegan un total de cuatro pasos con sus correspondientes subpasos en cada caso. La tabla 2 muestra los pasos y subpasos identificados en el M2.

\section{TABLA 2}

Modelo de análisis de los movimientos retóricos en reseñas de formación

\begin{tabular}{l} 
M2: VALORA EL LIBRO \\
P1: utiliza juicios de valor \\
\hline SP1A: Se refiere a la utilidad del libro para la comunidad académica \\
\hline SP1B: Realiza comentarios de valor sobre el contenido \\
\hline SP1C: Realiza comentarios de valor sobre la organización \\
\hline SP1D: Realiza un contraste con otros libros de similar temática \\
\hline P2: se refiere a la originalidad del libro \\
\hline P3: identifica al o los destinatarios ideales del libro \\
\hline P4: recomienda el libro \\
\hline SP4A: Especifica si recomienda o no el libro \\
\hline SP4B: Presenta un comentario a modo de justificación de la recomendación
\end{tabular}

La tabla 2 muestra que el M2 se divide en cuatro pasos, entre los cuales el P1 y el P4 se despliegan, a su vez, en una serie de subpasos. Como se ha señalado previamente en el MI, la distinción entre el P8 del M1 y algunos subpasos del P1 del M2 ha tenido que ser fijada mediante las características lingüísticas de cada uno.

En el primer paso identificado, el P1, uno de los pasos más recurrentes en el 57,3\% de las reseñas, el autor de la reseña emite juicios favorables o desfavorables sobre los contenidos o la organización del libro. Asimismo, mediante esos juicios puede valorar cuál es la utilidad del libro para la comunidad académica o compararlo con otros libros de la misma temática para diferenciarlo de estos positiva o negativamente. Un ejemplo ilustrativo:

(10) En conclusión este libro es una guía muy didáctica [sic] donde resuelve dudas gramaticales con explicaciones claras y sencillas y multiples [sic] ejercicios para hacer. Resulta fácil de usar ya que esta [sic] organizado en forma de ficha para ver las ideas mas [sic] claras. (Informante 3.57) 
En el P2, el menos recurrente con una aparición del 7,3\% de los textos analizados, se resaltarán qué aspectos son novedosos respecto a otros libros que se han escrito sobre la misma temática. Por ejemplo:

(11) A pesar de ello, añade una novedad con respecto a la participación del estudiante, es éste [sic] quién [sic] a veces compartirá o rebatirá lo que el temario proponga. (Informante 4.80)

En el tercer paso, el P3, el más recurrente de todos los pasos del M2 con el 79,1\% de la totalidad del corpus, se especula sobre cuál es el destinatario ideal de un libro de las características que se han definido. Un caso concreto:

(12) Este libro podría ser de gran utilidad (como pone en la presentación de este) para alumnos y profesores en el ámbito [sic] no universitario y para estudiantes de Filología. Tambien [sic] para todos aquellos que estén [sic] interesados en la gramatica [sic], sus usos y el funcionamiento. (Informante 2.36)

Para finalizar, el cuarto paso, el P4, también muy poco recurrente con un porcentaje del 8,3\%, se centra en el destinatario de la reseña. El autor se dirige directamente al lector especificando si le recomienda o no el libro, lo que puede ir acompañado de una justificación o de un comentario valorativo a modo global del libro. Véase el siguiente ejemplo:

(13) Es recomendable su lectura por el hecho de que profundiza sus explicaciones en áreas como las clases de palabras (sustantivo, adjetivo, verbo, adverbio...), las oraciones y grupos, la fonética y la fonología y la ortografía junto con la acentuación, puntuación, etc. (Informante 1.13)

Este último paso, el P4, es tan poco frecuente en las reseñas académicas de formación de este corpus que se plantea la posibilidad de que, por medio del P3, los estudiantes hayan considerado que la recomendación queda implícita al especificar cuál es el lector ideal. Por otra parte, pese a que la recomendación, tal y como señalan Babaii y Ansary (2007), tiene una función persuasiva que difiere de la función de marcar el posicionamiento del autor en la valoración, cabe mencionar que esta se ha comprendido como un paso del M2 en lugar de como un MR porque en este corpus no cumple esa función persuasiva.

Un análisis más pormenorizado de estos resultados, al igual que en el Mı, permite identificar qué pasos utilizan los informantes de manera obligatoria, de manera opcional o de manera poco frecuente, siguiendo los mismos criterios que en el caso anterior. En el M2, solo se constituye como paso obligatorio el P3 (identifica al o los destinatarios ideales del libro) y como paso opcional el P1 (utiliza juicios de valor). El P2 (se refiere a la originalidad del libro) y el P4 (recomienda el libro) tienen un índice de recurrencia muy bajo que los sitúa como pasos poco frecuentes.

\section{Conclusión}

En este estudio se ha caracterizado el género reseña académica de formación a partir de un corpus de 96 textos producidos en español por estudiantes del grado en Lenguas Modernas. 
Con ese propósito general, se ha aplicado el método de análisis de género para identificar los movimientos retóricos, los pasos y los subpasos utilizando como referencia el análisis de género (Swales, 1990, 2004) y, en concreto, la metodología de análisis BCU (Biber, Connor y Upton, 2007). De estos análisis, ha surgido y se ha validado un modelo de análisis de la configuración retórico-lingüística de las reseñas académicas de formación.

Este modelo analítico, validado para el análisis de las reseñas académicas de formación, se distancia claramente de las propuestas previas de modelos analíticos referidos a la reseña académica (Giammatteo y Ferrari, 2000; Motta-Roth, 2001; De Carvalho, 2002; Castro de CastiIlo, 2005; Díaz-Blanca, 2015), lo cual pone de manifiesto la incidencia del contexto en el que se ha producido este género y, al mismo tiempo, la necesidad de que los géneros producidos por estudiantes universitarios sean caracterizados. Asimismo, la propia configuración del modelo analítico en este contexto pone de relieve su valía como una herramienta útil para la alfabetización académica de los estudiantes de grado.

Por otra parte, en cuanto al modelo analítico que emerge de la praxis de los estudiantes, se constata en su configuración el lugar destacado que ocupa el Mı (“describe el libro"). Esto se puede observar en el mayor número de objetivos comunicativos que se despliegan para su desarrollo, y también en su recurrencia de uso, que es prácticamente de la totalidad del corpus. En este sentido, cabe destacar la alta recurrencia de pasos como el P1 ("presenta al autor del libro"), el P5 (“identifica el o los objetivos del libro”), el P6 (“describe la organización del libro") y el P8 ("se refiere a otras características del libro").

No sucede lo mismo con el M2, el cual se configura de manera menos matizada que el anterior mediante valoraciones que se refieren a aspectos marginales del libro reseñado que no son significativas para el propósito comunicativo global del texto. Esta escasa habilidad en el manejo de las competencias argumentativas viene a coincidir con los resultados del estudio de Castro y Sánchez (2013) acerca de la expresión de la opinión por parte de los estudiantes universitarios de grado, y pone de manifiesto la necesidad de que se analicen en el aula textos de expertos para hacer evidente los modos que existen en la disciplina de posicionarse críticamente, de construir una opinión de manera fundamentada, de incorporar otras voces, etc.

Conviene poner en relieve que este modelo analítico se extrae de un trabajo empírico que emerge de las prácticas escritas de la totalidad de estudiantes que completan un programa de formación, ya que esto permite destacar su valía como instrumento para constatar las transformaciones de los escritos de los estudiantes. En este caso, de este modelo se desprenden una serie de pautas que se pueden sistematizar en:

- Selección de los contenidos: qué pasos y subpasos del modelo aparecen y cuáles no.

- Importancia de los contenidos: qué pasos destacan con un mayor nivel de desarrollo.

- Organización de las ideas: qué jerarquización siguen los pasos y los subpasos. 
- Objetivos comunicativos del texto: describir, valorar o ambos.

- Nivel de desarrollo de cada movimiento retórico: estrategias y mecanismos para la descripción y la argumentación.

Por último, el propio desarrollo de la investigación y el modelo analítico resultante de esta plantean interrogantes en torno al nivel de experticia con el que los estudiantes podrán Ilevar a cabo una tarea de la complejidad del trabajo fin de grado si presentan dificultades para producir una reseña académica de formación. Asimismo, el modelo analítico plantea interesantes implicaciones didácticas en dos sentidos: como herramienta de evaluación y como herramienta de aprendizaje. Por una parte, como herramienta de evaluación, sirve de guía para que el docente, mediante la corrección de diferentes borradores, identifique los avances que realizan los estudiantes en la producción de la reseña midiendo parámetros de escritura como los anteriormente mencionados.

Por otra parte, como herramienta de aprendizaje, contribuye a la progresiva integración del estudiante en la comunidad discursiva mediante la lectura y el análisis crítico de textos propios de su disciplina. No obstante, este género solo se debe considerar como un modo de iniciar al estudiante en la cultura disciplinar, de que se sumerja en el lenguaje académico, ya que también tiene limitaciones relativas a (1) la extensión del texto, la cual se aleja de la propia de otros textos académicos más largos y segmentados en capítulos o secciones que exigen al estudiante sostener una orientación argumentativa, o (2) la incorporación del conocimiento ajeno en el texto.

\section{Bibliografía citada}

Alcaraz, María Ángeles, y Françoise Ssalager-Meyer, 2005: "Las reseñas de libros en español: estudio retórico y diacrónico", Spanish in Context 2 (1), 29-49.

AL-KHASAWNEH, Fadi Maher, 2017: "A genre analysis of research article abstracts written by native and non-native speakers of English", Journal of Applied Linguistics and LAnguage Research 4 (1), I-13.

AlzARI, Irina, 2012: "La reseña como género de formación en la carrera de Historia" en V Congreso Internacional de Letras, Buenos Aires: Facultad de Filosofía y Letras de la Universidad de Buenos Aires, 148-154.

Alzarl, Irina, Julián D’AlesSANDRo y Maricel RadiminskI, 2014: "La reseña de formación en historia" en Federico Navarro: Manual de escritura para carreras de humanidades, Buenos Aires: Facultad de Filosofía y Letras de la Universidad de Buenos Aires, 287-302.

BABAll, Esmat, y Hasan AnsARY, 2007: "A cross-disciplinary study of academic book reviews: defining a genre" en Alexander PAstukhov: Genres and text types in academic and media discourse, Oriol: Kartush Publisher, 127-141. 
Bajtín, Mijail, 1982: "El problema de los géneros discursivos" en Mijail BAjtín: Estética de la creación verbal, México: Siglo XXI, 248-293.

BathiA, Vijay Kumar, 1993: Analysing Genre: Language Use in Professional Settings, Londres: Routledge: Taylor \& Francis Group.

Biber, Douglas, Ulla Connor y Thomas Albin Upton, 2007: Discourse on the move. Using corpus analysis to describe discourse structure, Amsterdam: John Benjamins Publishing Company.

CAmps, Anna, y Montserrat CAStello, 2013: "La escritura académica en la universidad", Revista de docencia universitaria (REDU) 11 (1), 17-36.

Castro de Castillo, Esther, 2005: "La reseña" en Liliana Cubo de Severino: Los textos de la ciencia, Córdoba (Argentina): Comunicarte, 167-188.

Castro, María Cristina, y Martín Sánchez, 2013: "La expresión de opinión en textos académicos escritos por estudiantes universitarios", Revista Mexicana de Investigación Educativa 18 (57), 483-506.

De Carvalho, Gisele, 2002: "Resenhas acadêmicas e sua organização retórica", Revista Letras 57, $175-194$.

Diafabio de Anglat, Hilda Emilia, y Guadalupe Álvarez, 2019: "Estrategias retóricas del capítulo introductorio de tesis doctorales en Educación", Íkala, Revista de Lenguaje y Cultura 24 (1), 69-84.

Díaz-BlanCA, Lourdes, 2015: "Configuración retórica de las reseñas académicas", Letras 56 (91), 21-46.

DING, Huiling, 2007: "Genre analysis of personal statements: analysis of moves in application essays to medical and dental schools", English for Specific Purposes 26 (3), 368-392.

FerRaRI, Laura, 2005: "¿Cómo se escribe una reseña crítica?" en Graciela Vazquez: Español con fines académicos: de la comprensión a la producción de textos, Madrid: Edinumen, 29-44.

GeA-VALOR, M. Lluïsa, 2000: A pragmatic approach to politeness and modality in the book review articles, Valencia: Universitat de Valencia.

Giammatteo, Mabel, y Laura Ferrari, 2000: "La reseña crítica como clase textual: caracterización empírica y propuesta pedagógica", Anuario de Lingüística Hispánica XV-XVI, 1-15.

GIAnNonI, Davide Simone, 2009: "Negotiating research values across review genres: a case study in Applied Linguistics" en Ken HyLand y Giuliana Dianl: Academic evaluation. Review genres in university settings, Nueva York: Palgrave Macmillan, 17-33. 
Gómez, Leonardo, 1998: Gramática didáctica del español, Madrid: SM.

Groom, Nicholas, 2009: "Phraseology and epistemology in academic book reviews: a corpusdriven analysis of two humanities disciplines" en Ken HrLand y Giuliana Dianl: Academic evaIuation. Review genres in university settings, Nueva York: Palgrave Macmillan, 122-139.

Hammond, John, 2011: "Classroom discourse" en Ken Hyland y Brian Paltridge: The continnum companion to discourse analysis, Londres: Continuum International Publishing Group, 291-305.

HyLAND, Ken, 2007: Disciplinary discourses: social interactions in academic writing, Michigan: Ann Arbor: University of Michigan Press.

HyLAND, Ken, 2014: "Disciplinary discourses: stance in research articles" en Christopher CandLIN y Ken HyLand: Writing: texts, processes and practices, Nueva York: Routledge, 99-121.

Kanoksilpatham, Budsaba, 2007: "Introduction to move analysis" en Douglas Biber, Ulla Connor y Thomas Albin Upton: Discourse on the move. Using corpus analysis to describe discourse structure, Amsterdam: John Benjamins Publishing Company, 23-41.

Maswana, Sayako, Toshiyuki Kanamarub y Akira Tajinob, 2015: "Move analysis of research articles across five engineering fields: What they share and what they do not", Ampersand 2, 1-11.

МоттA-Rотн, Desiree, 1995: "Book reviews and disciplinary discourse: defining a genre" en Teachers of English to Speakers of Other Languages (TESOL) - 29th Annual Convention \& Exposition, Long Beach (California), 385-386.

Мотта-Rотн, Desiree, 1996: "Same genre,different discipline: a genre-based study of book reviews in academe", The ESPecialist 17 (2), 99-131.

Мотта-Rотн, Desiree, 1998: "Discourse analysis and academic book reviews: a study of text and disciplinary cultures" en Inmaculada Fortanet, Santiago Posteguillo, Juan Cartlos Palmer y Juan Francisco Coll: Genre studies in English for Academic Purposes, Castelló: Universitat Jaume I, 29-58.

Мотта-Rотн, Desiree, 2001: "A construção social do gênero resenha acadêmica", Trabalhos Em Lingüística Aplicada 38, 29-45.

Navarro, Federico, 2011: Análisis histórico del discurso. La evaluación en las reseñas del Instituto de Filología de Buenos Aires (1939-1989). Tesis de doctorado, Universidad de Valladolid. 
Navarro, Federico, y Abramovich, Ana Luz, 2012: "La reseña académica" en Lucía Natale: En carrera: escritura y lectura de textos académicos y profesionales, Polvorines (Argentina): Universidad Nacional de General Sarmiento, 39-60.

North, Stephen, 1992: "On book reviews in rhetoric and composition", Rhetoric Review $10(2), 348-363$.

Piqué-Noguera, Carmen, y Ramón Camaño-Puig, 2015: "El resumen del artículo de investigación: análisis del género en un corpus de textos de Enfermería", Signos 48 (87), 77-94.

Pramoolsook, Issra, y Li QIAN, 2013: "Comparative Genre Analysis of English Argumentative Essays Written by Chinese English and Non-English Major Students", Arab World English Journal 4 (1), 213-223.

SABAJ, Omar, 2012: "Uso de movidas retóricas y patrones léxico-gramaticales en artículos de investigación en español. Implicancias para la enseñanza de la escritura científica", Boletín de Filología XLVII (1), 165-186.

Sabaj, Omar, Paulina Toro y Miguel Fuentes, 2011: "Construcción de un modelo de movidas retóricas para el análisis de artóculos de investigación en español", Onomázein 24, 245-271.

Salmani, Mohammad Ali, y Hamed Montazeran, 2012: "The book review genre: A structural move analysis", International Journal of Language Studies 6 (1), 1-30.

Samraj, Betty, 2008: "A discourse analysis of master's theses across disciplines with a focus on introductions", Journal of English for Academic Purposes 7 (1), 55-67.

Swales, John, 1990: Genre Analysis, Cambridge: Cambridge University Press.

SwaLes, John, 2004: Research genres: explorations and applications, Cambridge: University of Cambridge.

VelAsco, Erlantz, 2017: El trabajo fin de grado en la Universidad de Deusto: una aproximación a su composición retórico-discursiva y a las representaciones sociales desde la perspectiva de sus autores. Tesis de doctorado, Universidad de Deusto (Bilbao).

Venegas, René, Sofía Zamora y Amparo Galdames, 2016: "Hacia un modelo retórico-discursivo del macrogénero trabajo final de grado en Licenciatura", Signos 49 (1), 247-279.

Zamora, Sofía, y René VeneGas, 2012: "Estructura y propósitos comunicativos en tesis de magíster y licenciatura", Literatura y Lingüística 27, 201-218. 


\section{Anexo}

\section{FIGURA 1}

Instrumento definitivo de creación del corpus

La Biblioteca de la UD quiere incorporar en su catálogo una reseña de los libros de reciente incorporación.

\section{¿̄Nos echas una mano?}

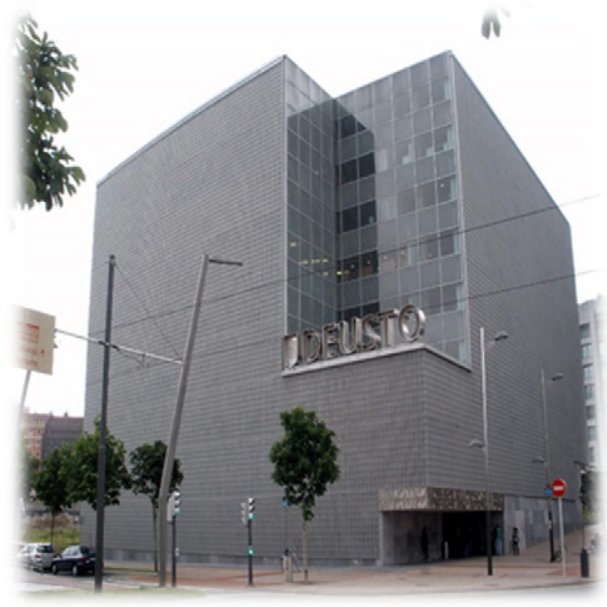

Necesitamos que escribas un texto de no menos de 200 palabras (máx. 300). TEN EN CUENTA QUE...

Este texto tiene como finalidad informar a los usuarios, estudiantes, que vayan a la biblioteca para saber si es el libro que están buscando.

Por ello, el texto que escribas debe incluir los aspectos del libro que consideres oportunos. La siguiente lista puede servir de ayuda: autor o autores, tema, organización del libro y contenidos, a quién puede interesar, valoración sobre su utilidad para la comunidad académica...

Nosotros te facilitaremos la portada, la contraportada, la presentación y el índice.

\section{¡COLABORA CON NOSOTROS!}

